\title{
Sliding mode control techniques for combined energy and attitude control system
}

\begin{abstract}
Combined Energy and Attitude Control System (CEACS) is an optimization approach that combines the energy storage system and the attitude control system. With a double counter rotating flywheel simultaneously serving as energy storage device and as attitude control actuator, CEACS requires an accurate control strategy to obtain the mission requirements. In addition, it is important to design the control law to be invariant to uncertainties and disturbances, and guarantee robustness as CEACS inherits these in-orbit uncertainties. This paper presents a nonlinear control employing sliding mode to enhance the CEACS attitude control capability. The mathematical model for the conventional and boundary layer sliding mode controls are developed herein for CEACS. The controller provides enhancement in pointing accuracies, reasonable transient responses and a robustness against uncertainties and in-orbit disturbances.
\end{abstract}

Keyword: Attitude control; CEACS; Flywheel; Sliding mode control 